\title{
HNF-4 $\alpha$ downregulation promotes tumor migration and invasion by regulating E-cadherin in renal cell carcinoma
}

\author{
YAOHUI GAO $^{1 *}$, YANG YAN $^{2 *}$, JING GUO $^{1}$, QIAN ZHANG $^{1}$, DEXI BI $^{1}$, FEN WANG $^{2,3}$, \\ ZHENGYAN CHANG ${ }^{1}$, LING LU $^{1}$, XUDONG YAO $^{2}$ and QING WEI $^{1}$ \\ Departments of ${ }^{1}$ Pathology, ${ }^{2}$ Urology and ${ }^{3}$ Anesthesiology, Shanghai Tenth People's Hospital, \\ Tongji University, Shanghai 200072, P.R. China
}

Received October 5, 2018; Accepted June 18, 2019

DOI: 10.3892/or.2019.7214

\begin{abstract}
Renal cell carcinoma (RCC) is the most common malignant disease of the kidneys in adults. Patients with metastatic RCC have an unusually poor prognosis and exhibit resistance to all current therapies. Therefore, it is necessary to explore novel molecules involved in the progression of RCC and to identify effective therapeutic targets. Hepatocyte nuclear factor- $4 \alpha$ (HNF-4 $\alpha$ ) serves an important role in hepatocyte differentiation and is involved in the progression of liver cancer; however, the functional role of HNF-4 $\alpha$ has not been well established in RCC. The present study reported that HNF-4 $\alpha$ expression was markedly downregulated in RCC tissue samples compared with in normal controls by immunohistochemistry and RNA-sequencing analysis. Statistical analysis demonstrated that $\mathrm{HNF}-4 \alpha$ downregulation was significantly associated with tumor stage, recurrence, metastasis and poor prognosis in patients with RCC. Furthermore, wound-healing and Transwell assays revealed that downregulation of $\mathrm{HNF}-4 \alpha$ promoted cell migration and invasion by transcriptionally regulating E-cadherin in RCC. Finally, a positive correlation was revealed between HNF- $4 \alpha$ expression and E-cadherin expression, and patients with low E-cadherin expression also had a poor prognosis. These findings may provide novel insights into the biological effects of HNF- $4 \alpha$ and lay the foundation for the discovery of molecular therapeutic targets in RCC.
\end{abstract}

Correspondence to: Dr Qing Wei, Department of Pathology, Shanghai Tenth People's Hospital, Tongji University, 301 Yanchang Middle Road, Shanghai 200072, P.R. China

E-mail: weiqing1971@126.com

Professor Xudong Yao, Department of Urology, Shanghai Tenth People's Hospital, Tongji University, 301 Yanchang Middle Road, Shanghai 200072, P.R. China

E-mail: yaoxudong67@sina.com

*Contributed equally

Key words: renal cell carcinoma, hepatocyte nuclear factor- $4 \alpha$, E-cadherin, migration, invasion

\section{Introduction}

Renal cell carcinoma (RCC) is one of the most common malignant diseases of the kidney, which accounts for 2-3\% of cases among all types of cancer (1). In Western countries, RCC has risen by $\sim 2 \%$ in the last two decades (2). In 2016, kidney cancer accounted for an estimated 62,700 new cancer diagnoses and 14,240 cases of mortality in the USA (3). To date, the main treatment for RCC is complete or partial surgical resection combined with chemotherapy or radiotherapy. Metastatic RCC displays a poor response to chemotherapy and radiotherapy, due to systemic toxicity and increased expression of multidrug resistance genes; this is responsible for the high mortality rate of advanced RCC $(4,5)$. The 5-year overall survival rate of patients with metastatic RCC is $<10 \%$ and the median survival time is only 1.5 years (6). In addition, $20-25 \%$ of patients with RCC have already reached the metastatic phase upon initial diagnosis (7). Therefore, there is an urgent need to explore novel molecules involved in the progression of RCC, in order to identify therapeutic targets for patients with RCC.

Hepatocyte nuclear factor- $4 \alpha$ (HNF- $4 \alpha$ ) is one of the major transcription factors that regulate liver cell differentiation. The expression of HNF- $4 \alpha$ is tissue-specific, and it is highly expressed in the liver, kidneys, intestines and pancreas (8). In the liver, HNF- $4 \alpha$ regulates the expression of a series of genes involved in the metabolism of amino acids, lipids, carbohydrates and cholesterol, the transport of ions, blood coagulation and cell proliferation (9-11). In addition, HNF-4 $\alpha$ is involved in cell differentiation and maintenance of epithelial morphology in certain cell types $(12,13)$. At present, research into HNF-4 $\alpha$ in cancer has primarily focused on liver cancer. The transcriptional activity of HNF- $4 \alpha$ is decreased or completely lost in $\sim 70 \%$ of patients with liver cancer, and the prognosis of patients with low expression of HNF- $4 \alpha$ is poor (14). In addition, HNF- $4 \alpha$ has been reported to be associated with the malignancy of liver cancer in mouse models; in a previous study, upregulation of HNF- $4 \alpha$ expression was able to transform invasive liver cancer into a less malignant phenotype (15). These data are indicative of an important role of HNF- $4 \alpha$ inactivation in the progression of liver cancer. The loss of functional activity of HNF- $4 \alpha$ has also been described in RCC (16); therefore, it is of interest to analyze whether HNF- $4 \alpha$ has a functional role in RCC tumorigenesis and progression. 
Tumor metastasis is an important factor in evaluating prognosis and predicting therapeutic response. Epithelial to mesenchymal transition (EMT) is an important process during cellular transformation, by which epithelial tumor cells lose their polarity, rearrange cytoskeletal elements, and display reduced intercellular adhesion and increased motility, endowing cancer cells with invasive and metastatic properties (17). A critical molecular feature of EMT is the downregulation of E-cadherin, which is a cell adhesion molecule present in the majority of normal epithelial cell membranes. E-cadherin is considered to act as a tumor suppressor inhibiting metastasis in various types of cancer, including hepatocellular carcinoma, head and neck carcinoma, squamous cell carcinoma of the skin and esophagus, and melanoma (18). Re-expression of E-cadherin is sufficient to reduce the aggressiveness of tumor cells. Conversely, E-cadherin depletion leads to mesenchymal morphology, and increased cell migration and invasion (19).

The present study aimed to investigate whether HNF- $4 \alpha$ may serve as a molecular marker for RCC migration and invasion. In the present study, reduced expression of $\mathrm{HNF}-4 \alpha$ promoted tumor migration and invasion by regulating E-cadherin in RCC. The present results highlighted the potential role of HNF-4 $\alpha$ as a therapeutic target for the treatment of RCC.

\section{Materials and methods}

Cell culture and treatment. Human RCC cell lines A498 and OS-RC-2, and the normal kidney cell line HK2 were obtained from the Cell Bank of the Chinese Academy of Sciences. 293T cells were obtained from Dr Yong Zhang (Rui-Jin Hospital). The cells were cultured in Dulbecco's modified Eagle's medium (Sigma-Aldrich; Merck KGaA) supplemented with $10 \%$ fetal bovine serum (Gibco; Thermo Fisher Scientific, Inc.). All cell lines were cultured in a humidified atmosphere containing $5 \% \mathrm{CO}_{2} / 95 \%$ air at $37^{\circ} \mathrm{C}$.

Clinical tissue samples. A total of 30 primary RCC tissues, 18 metastatic tissues and 22 adjacent normal tissues were obtained from primary RCC patients, which were histopathologically diagnosed between June 2015 and August 2018. The patients were aged between 56 and 77 years old (mean age, 68.5 years), and included 27 men and 21 women. All samples obtained were primary tumors and patients were untreated prior to surgery. Tissues were collected from the Shanghai Tenth People's Hospital Affiliated to Tongji University School of Medicine. The present study was approved by the Medical Ethical Committee of Shanghai Tenth People's Hospital. All patients provided written informed consent prior to participation and agreed to publication of the present study.

RNA-sequencing analysis. The mRNA expression data of HNF- $4 \alpha$ and E-cadherin were obtained from The Cancer Genome Atlas (TCGA); these data were obtained from 1,019 tumor tissues and 139 adjacent normal tissues from patients with RCC. TCGA dataset used in this study was retrieved from the Genomic Data Commons (GDC) Data Portal (portal. gdc.cancer.gov). The identifiers of the cases included in this study are listed in Table SI. All data used in this study are readily accessible via these identifiers on the GDC Data Portal website. To segregate patients with RCC according to high or low expression, the median expression was calculated. If expression was under the median value, patients were included in the low group, and vice versa.

Immunohistochemistry (IHC). Briefly, tissue samples (length, $<3 \mathrm{~mm}$ ) were fixed with $4 \%$ paraformaldehyde for $24 \mathrm{~h}$ at room temperature, dehydrated through a graded alcohol series and embedded in paraffin. Tissue slides $(4 \mu \mathrm{m})$ were then deparaffinized at $60^{\circ} \mathrm{C}$, followed by treatment with $100 \%$ xylene for $20 \mathrm{~min}$ and a graded series of ethanol at room temperature. The slides were incubated in a $3 \%$ hydrogen peroxide solution for $15 \mathrm{~min}$ at room temperature, followed by incubation in $10 \mathrm{mM}$ sodium citrate buffer at $95^{\circ} \mathrm{C}$ for $10 \mathrm{~min}$. Subsequently, the slides were rinsed in PBS and incubated with 5\% BSA (Sigma-Aldrich; Merck KGaA) for $30 \mathrm{~min}$ at room temperature. The slides were then incubated with primary antibodies at $4{ }^{\circ} \mathrm{C}$ overnight. The following antibodies were used: HNF-4 $\alpha$ (1:50, cat. no. ab201460; Abcam) and E-cadherin (1:100, cat. no. 24E10; Cell Signaling Technology, Inc.). The slides were visualized using the standard avidin-biotinylated peroxidase complex method. Briefly, biotinylated secondary antibody (100 $\mu 1,1: 2,000$, cat. no. ab6720; Abcam) was applied to the sections on the slides and the slides were incubated in a humidified chamber at room temperature for $30 \mathrm{~min}$ A DAB Substrate kit (cat. no. JM3363; Shanghai Youyu Biotech Co., Ltd.) was used for staining, according to the manufacturer's protocol. Finally, 10\% hematoxylin was used for counterstaining at $37^{\circ} \mathrm{C}$ for $3 \mathrm{~min}$ and morphological images were observed under an Olympus BX51 microscope. All staining was blindly scored by two pathologists according to the intensity of staining: No staining, 0 ; weak staining, 1 ; moderate staining, 2; strong staining, 3, and the area of stained cells: $0 \%, 0 ; 1-24 \%, 1 ; 25-49 \%, 2 ; 50-74 \%, 3 ; 75-100 \%, 4$. The final immunoreactive score was determined by multiplying the intensity score with the area of stained cells; scores ranged between 0 (the minimum score) and 12 (the maximum score).

Reverse transcription-quantitative polymerase chain reaction (RT-qPCR). Total RNA was isolated using TRIzol ${ }^{\circledR}$ reagent (Invitrogen; Thermo Fisher Scientific, Inc.) and treated with RNase-free DNase (Promega Corporation). RT was performed using PrimeScript 1st Strand cDNA Synthesis kit (Takara Biotechnology Co., Ltd.) according to the manufacturer's protocol. qPCR was carried out with SYBR-Green PCR Master Mixture Reagent (Applied Biosystems; Thermo Fisher Scientific, Inc.) using an ABI 7500 Detection system (Applied Biosystems; Thermo Fisher Scientific, Inc.). Thermocycling conditions were as follows: An initial cycle at $95^{\circ} \mathrm{C}$ for $10 \mathrm{~min}$, followed by 35 cycles of denaturation at $95^{\circ} \mathrm{C}$ for $30 \mathrm{sec}$, annealing at $60^{\circ} \mathrm{C}$ for $30 \mathrm{sec}$ and extension at $72^{\circ} \mathrm{C}$ for $30 \mathrm{sec}$, and a final extension step at $72^{\circ} \mathrm{C}$ for $10 \mathrm{~min}$ to complete the amplification. The fluorescence threshold value was calculated using 7500 software v2.0.6 (Applied Biosystems; Thermo Fisher Scientific, Inc.). The final quantification was determined using the $2^{-\Delta \Delta C q}$ method (20). Experiments were repeated at least three times with similar results. A list of qPCR primers is presented in Table SII.

Plasmids, short hairpin (shRNA) and viral infection. Human HNF- $4 \alpha$ and E-cadherin cDNA amplified from $293 \mathrm{~T}$ 
cells were cloned and inserted into a pLVX-Puromycin lentiviral expression vector (Clontech Laboratories, Inc.). Complementary shRNA oligonucleotides against HNF- $4 \alpha$ and E-cadherin were synthesized, annealed and ligated into pSIREN-RetroQ, according to the manufacturer's protocol (Clontech Laboratories, Inc.). The sequence for E-cadherin shRNA was 5'-AAGATAGGAGTTCTCTGATGC-3' and the sequence for HNF- $4 \alpha$ shRNA has been described previously (5). These plasmids (500 ng) were co-transfected into 293T cells $\left(5 \times 10^{5}\right.$ cells/well) using $4 \mu 1$ FuGene 6 (Roche Diagnostics) with packaging plasmids, including psPAX2 and pMD2G for the lentivirus or VSV-G and gag-pol, the retrovirus, for $20 \mathrm{~min}$ at room temperature. The packaging plasmids were obtained from Dr Shaoming Shen (Shanghai Jiaotong University). After transfection for $48 \mathrm{~h}$, the viral supernatant $(1.5 \mathrm{ml} /$ well $)$ was collected, filter-sterilized and added to cells in a 6 -well plate $\left(1.5 \times 10^{5}\right.$ cells/well $)$ containing polybrene $(4 \mu \mathrm{g} / \mathrm{ml})$; puromycin $(1 \mu \mathrm{g} / \mathrm{ml})$ was added to select the stably infected cells after another $48 \mathrm{~h}$. The multiplicity of infection of viruses ranged between 10 and $50 \mathrm{PFU} /$ cell. In the control groups, cells were infected with empty vector (EV) or a non-specific control (NC). The sequence for NC shRNA was 5'-TTCTCCGAACGTGTCACG-3'. Selection was stopped as soon as the non-infected control cells died off.

Western blotting. Western blotting was performed as described previously (21). The following antibodies were used: Rabbit antibodies against HNF-4 $\alpha$ (cat. no. ab201460; Abcam), E-cadherin (cat. no. 24E10; Cell Signaling Technology, Inc.), $\mathrm{N}$-cadherin (cat. no. 13116; Cell Signaling Technology, Inc.), $\beta$-catenin (cat. no. 8480; Cell Signaling Technology, Inc.), phosphorylated (P)-p65 (cat. no. 3033; Cell Signaling Technology, Inc.), p65 (cat. no. 8242; Cell Signaling Technology, Inc.), P-STAT3 (cat. no. ab76315; Abcam), P-AKT (cat. no. ab38449; Abcam), AKT (cat. no. ab8805; Abcam) and $\beta$-actin monoclonal antibody (cat. no. HRP-60008; Proteintech Group, Inc.); mouse antibodies against STAT3 (cat. no. ab119352; Abcam).

Cell proliferation assay. Cell proliferation was evaluated using the Cell Counting kit-8 (CCK-8) assay (WST-8; Dojindo Molecular Technologies, Inc.). Briefly, $100 \mu 1$ cells were seeded into 96-well plates at a density of 2,000 cells/well on days 1 , 2 and 3. Subsequently, $10 \mu 1$ WST- 8 was added and cells were incubated at $37^{\circ} \mathrm{C}$ for $3 \mathrm{~h}$. Absorbance was measured at a wavelength of $450 \mathrm{~nm}$ using a Synergy H4 Hybrid Microplate Reader (BioTek Instruments, Inc.).

Wound-healing assay. Cells $\left(2 \times 10^{5}\right.$ cells/well) were seeded in 6-well plates. After starving overnight in medium supplemented with $1 \%$ FBS, the $>90 \%$ confluent monolayers were scraped with a $200-\mu 1$ pipette tip to create a linear wound. Plates were washed with PBS and cultured with complete medium for $24 \mathrm{~h}$. Images of the wounds were captured with a phase contrast light microscope (Olympus Corporation) and the horizontal distance between the edges of the wound was measured. This assay was performed in triplicate.

Transwell migration and invasion assay. For the Transwell migration assay, cells $\left(2 \times 10^{4}\right.$ cells/well) were suspended in $200 \mu 1$ serum-free Dulbecco's modified Eagle's medium (DMEM, Sigma-Aldrich; Merck KGaA) and plated on $8 \mu \mathrm{M}$
Transwell filters (Corning, Inc.) in 24-well plates containing $500 \mu \mathrm{l}$ DMEM supplemented with 10\% FBS. For the Transwell invasion assay, cells $\left(5 \times 10^{4}\right.$ cells/well) were suspended in $200 \mu \mathrm{l}$ serum-free DMEM and seeded on the upper chamber of Transwell filters in 24-well plates containing $500 \mu 1$ DMEM supplemented with $10 \%$ FBS, which was coated with Matrigel (BD Biosciences). The lower chamber was filled with $600 \mu \mathrm{l}$ complete DMEM. After $36 \mathrm{~h}$ at $37^{\circ} \mathrm{C}$, non-migratory or non-invasive cells on the upper side of the membrane were removed with a cotton swab, whereas cells on the lower side of the membrane were fixed with $100 \%$ methanol for $20 \mathrm{~min}$ and stained with $1 \%$ crystal violet for $15 \mathrm{~min}$ at $37^{\circ} \mathrm{C}$. Images of the cells were captured and counted in five random fields under a dissecting microscope. This assay was performed in triplicate.

Luciferase assay. Sequences in the E-cadherin promoter (2,000 bp) were amplified by PCR from the genomic DNA of 293T cells and subcloned into a pGL3-Basic vector (Promega Corporation) to construct luciferase reporter plasmids. For the luciferase assay, $293 \mathrm{~T}$ cells $\left(5 \times 10^{5}\right.$ cells/well) were seeded in a 6-well plate, and co-transfected with HNF- $4 \alpha$ expression vector (500 ng), luciferase reporter plasmids driven by promoter fragments of E-cadherin (500 ng), and pRLSV40-Renilla (50 ng) using $4 \mu \mathrm{l} \mathrm{FuGene} 6$ (Roche Diagnostics). A total of $36 \mathrm{~h}$ post-transfection, cells were lysed and analyzed using the Dual-Luciferase Assay system (cat. no. E1910; Promega Corporation) according to the manufacturer's protocol.

Statistical analysis. All experiments were repeated at least three times. All statistical analyses were evaluated using the GraphPad Prism 6.0 software (GraphPad Software, Inc.). The Kaplan-Meier method was used to analyze overall survival and comparisons were analyzed by log-rank test. The Pearson's $\chi^{2}$ test was used to evaluate associations. Spearman's rank correlation was used to evaluate the correlation between HNF- $4 \alpha$ and E-cadherin mRNA expression in different grades of RCC. One-way ANOVA followed by Bonferroni post hoc test was used for multiple comparisons. Unless otherwise described, comparisons between two groups were analyzed by Student's t-test (unpaired, two-tailed). $\mathrm{P}<0.05$ was considered to indicate a statistically significant difference.

\section{Results}

$H N F-4 \alpha$ is downregulated and associated with a poor prognosis in RCC. Immunohistochemical analysis of RCC and normal adjacent tissues demonstrated that there was a significant decrease in $\mathrm{HNF}-4 \alpha$ protein expression in RCC tissues compared with in normal tissues adjacent to the tumor tissues (Fig. 1A and B). In addition, HNF- $4 \alpha$ expression was significantly lower in metastatic tissues compared with in primary tumors (Fig. 1C). Furthermore, downregulation of HNF- $4 \alpha$ mRNA expression and the negative association between $\mathrm{HNF}-4 \alpha$ expression and pathological grade was confirmed in 1,158 RCC samples using TCGA database (Fig. 1D and E).

The present study further investigated whether HNF-4 $\alpha$ expression was associated with RCC patient prognosis. The results indicated that downregulation of HNF- $4 \alpha$ was significantly associated with tumor recurrence and metastasis in 
A
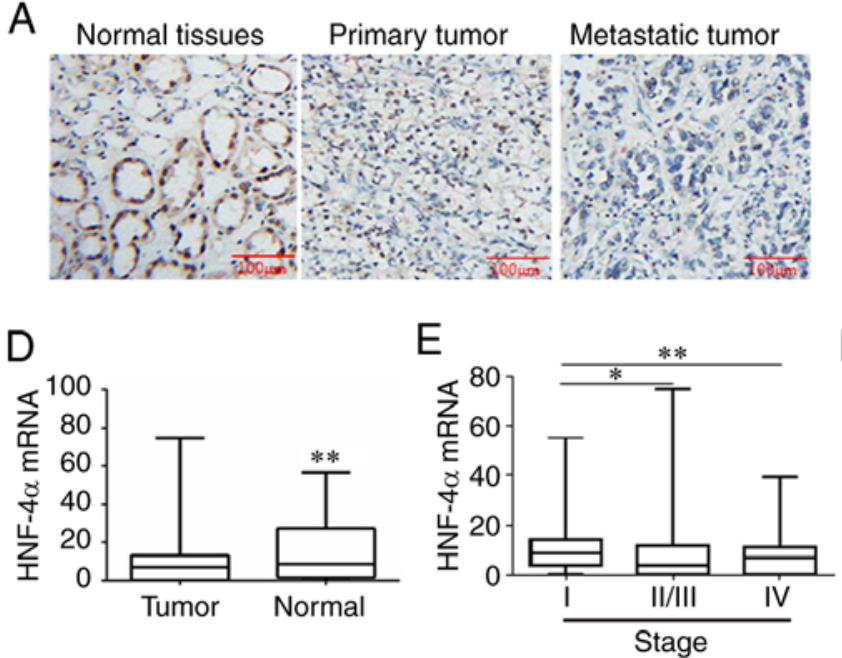

B
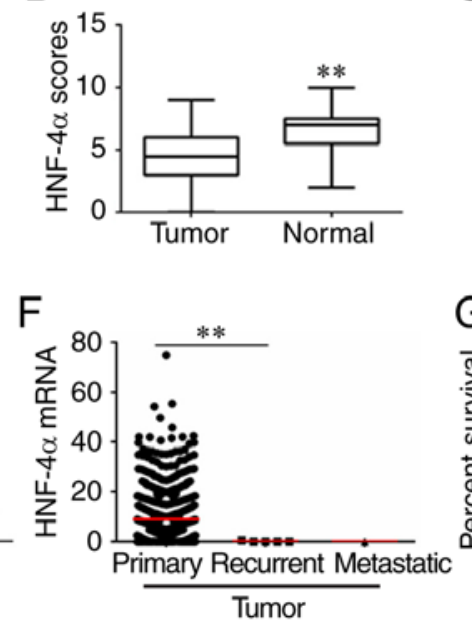

C
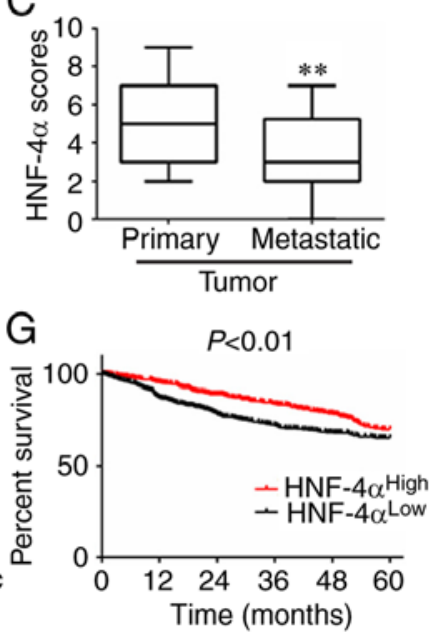

Figure 1. HNF-4 $\alpha$ is downregulated and associated with a poor prognosis in RCC. (A) Representative immunohistochemistry images of RCC samples for the expression of HNF-4 $\alpha$ protein. (B and C) HNF-4 $\alpha$ expression scores are presented as box plots, with the horizontal lines representing the median. The plots range between the minimum and maximum. $\mathrm{RCC}$ tissues $(\mathrm{n}=48)$ with adjacent normal tissues $(\mathrm{n}=22)$, and primary $(\mathrm{n}=30)$ and metastatic $(\mathrm{n}=18) \mathrm{RCC}$ tissues were compared. P-values were calculated via Student's t-test. Information on HNF-4 $\alpha$ expression in different pathological types and grades of RCC was derived from TCGA database. (D) Tumor $(n=1,019)$ and normal $(n=139)$ tissues were compared and analyzed using Student's t-test. (E) Stage I (n=473), II/III $(\mathrm{n}=390)$ and IV $(\mathrm{n}=117)$ RCC were compared and analyzed using one-way ANOVA followed by Bonferroni post hoc test. (F) Primary (n=1,013), recurrent $(\mathrm{n}=5)$ and metastatic $(\mathrm{n}=1)$ RCC tissues were compared and analyzed using one-way ANOVA followed by Bonferroni post hoc test. (G) Kaplan-Meier analysis of the association between HNF- $4 \alpha$ expression and survival time in patients with RCC; data were derived from TCGA database. Cases were classified into low and high expression groups. Results were analyzed by log-rank test. ${ }^{*} \mathrm{P}<0.05,{ }^{* *} \mathrm{P}<0.01$. HNF- $4 \alpha$, hepatocyte nuclear factor- $4 \alpha$; RCC, renal cell carcinoma; TCGA, The Cancer Genome Atlas.

RCC (Fig. 1F). The association of HNF-4 $\alpha$ expression with overall survival in patients with RCC was analyzed using TCGA database. The results revealed that patients with RCC with lower HNF- $4 \alpha$ expression had a relatively reduced 5-year survival compared with those with higher HNF-4 $\alpha$ expression, according to TCGA database (Fig. 1G). Taken together, these results suggested that HNF- $4 \alpha$ may serve as a prognostic marker of RCC progression.

$H N F-4 \alpha$ negatively regulates the migration and invasion of RCC cells. Tumor metastasis is an important indicator for evaluating the prognosis of patients. The present study investigated the effect of HNF- $4 \alpha$ on RCC cell migration and invasion. Since $\mathrm{HNF}-4 \alpha$ is positively regulated by von Hippel-Lindau (VHL) (14), HNF-4 $\alpha$ was overexpressed in VHL-deficient A498 cells and downregulated in VHL-proficient OS-RC-2 cells (Fig. 2A and B). The results revealed that the differences in $\mathrm{HNF}-4 \alpha$ expression had little effect on the proliferation of RCC cells (Fig. 2C and D). In addition, silencing $\mathrm{HNF}-4 \alpha$ in $\mathrm{HK} 2$, which is a proximal tubular cell line derived from normal kidney, did not affect cell proliferation (Fig. S1A).

Data from the wound-healing assay indicated that overexpression of $\mathrm{HNF}-4 \alpha$ significantly inhibited the migration of A498 cells, whereas HNF-4 $\alpha$ knockdown promoted OS-RC-2 cell migration (Fig. 2E and F). The results of the Transwell assay also indicated that HNF-4 $\alpha$ overexpression markedly suppressed A498 cell migration and invasion (Fig. 2G and I). Conversely, downregulation of $\mathrm{HNF}-4 \alpha$ significantly promoted the migration and invasion of OS-RC-2 cells (Fig. 2H and J). Furthermore, the Transwell migration assay revealed that HK2 cells did not migrate, even when the expression of HNF- $4 \alpha$ was silenced under the same experimental conditions as tumor cells (Fig. S1B). These results suggested that HNF- $4 \alpha$ negatively regulated the migration and invasion of RCC cells.

$H N F-4 \alpha$ regulates the migration and invasion of $R C C$ cells by E-cadherin. Tumor migration and invasion is regulated by various genes involved in different signaling pathways, including the EMT, JNK-STAT, NF- $\mathrm{B}, \mathrm{WNT} / \beta$-catenin and PI3K/AKT pathways (22-25). To explore whether HNF-4 $\alpha$ regulates cell invasion and migration via these known proteins and signaling pathways, the expression of a number of proteins that serve important roles in tumor migration- and invasion-related signaling pathways was assessed in cells with overexpression or knockdown of HNF-4 $\alpha$. It was observed that $\mathrm{HNF}-4 \alpha$ was able to regulate the expression of E-cadherin and $\mathrm{N}$-cadherin, which are involved in the EMT process, but had little effect on other proteins, including $\beta$-catenin, P-p65/p65, P-STAT3/STAT3 and P-AKT/AKT (Fig. 3A-D). Furthermore, it was confirmed that HNF-4 $\alpha$ was able to transcriptionally regulate the expression of E-cadherin, but not $\mathrm{N}$-cadherin, Vimentin and Twist in the EMT process (Fig. 3E and F).

Snail and Slug are known transcription factors that regulate E-cadherin. HNF-4 $\alpha$ did not transcriptionally regulate the expression of Snail and Slug in RCC cells (Fig. 3G and H). These results suggested that $\mathrm{HNF}-4 \alpha$ may directly regulate the expression of E-cadherin. A $2 \mathrm{~kb}$ DNA fragment upstream of the transcriptional start site of E-cadherin was subcloned into the luciferase reporter vector pGL3-basic, which was subsequently co-transfected into $293 \mathrm{~T}$ cells alongside a HNF-4 $\alpha$ expression vector plasmid and the internal control Renilla. Luciferase activity of the E-cadherin promoter was activated by ectopic expression of HNF-4 $\alpha$ (Fig. 3I and J). These results suggested that HNF-4 may bind to the promoter of E-cadherin to regulate its transcription. 
A

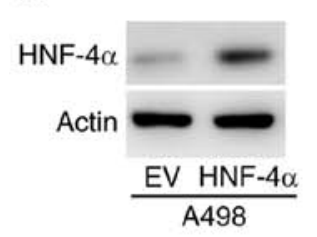

C

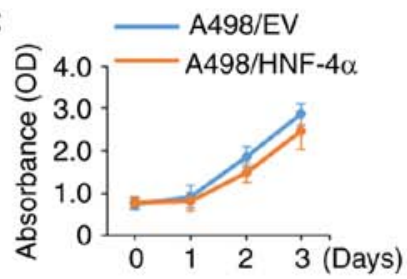

B

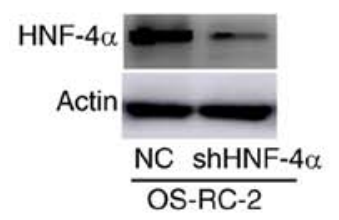

G
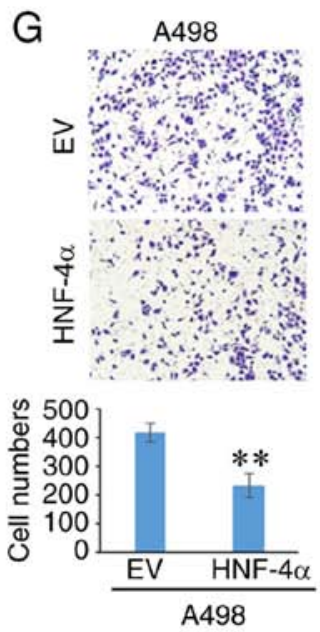

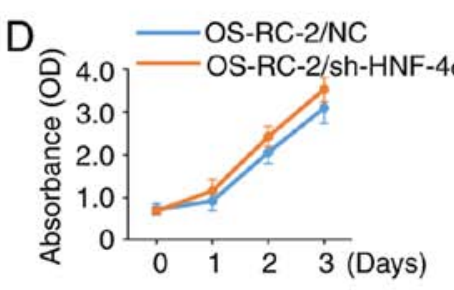

H OS-RC-2
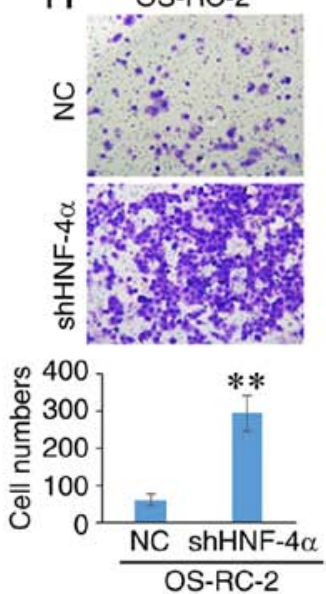

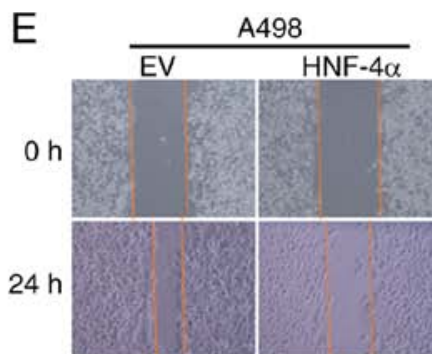

$\mathrm{F}$
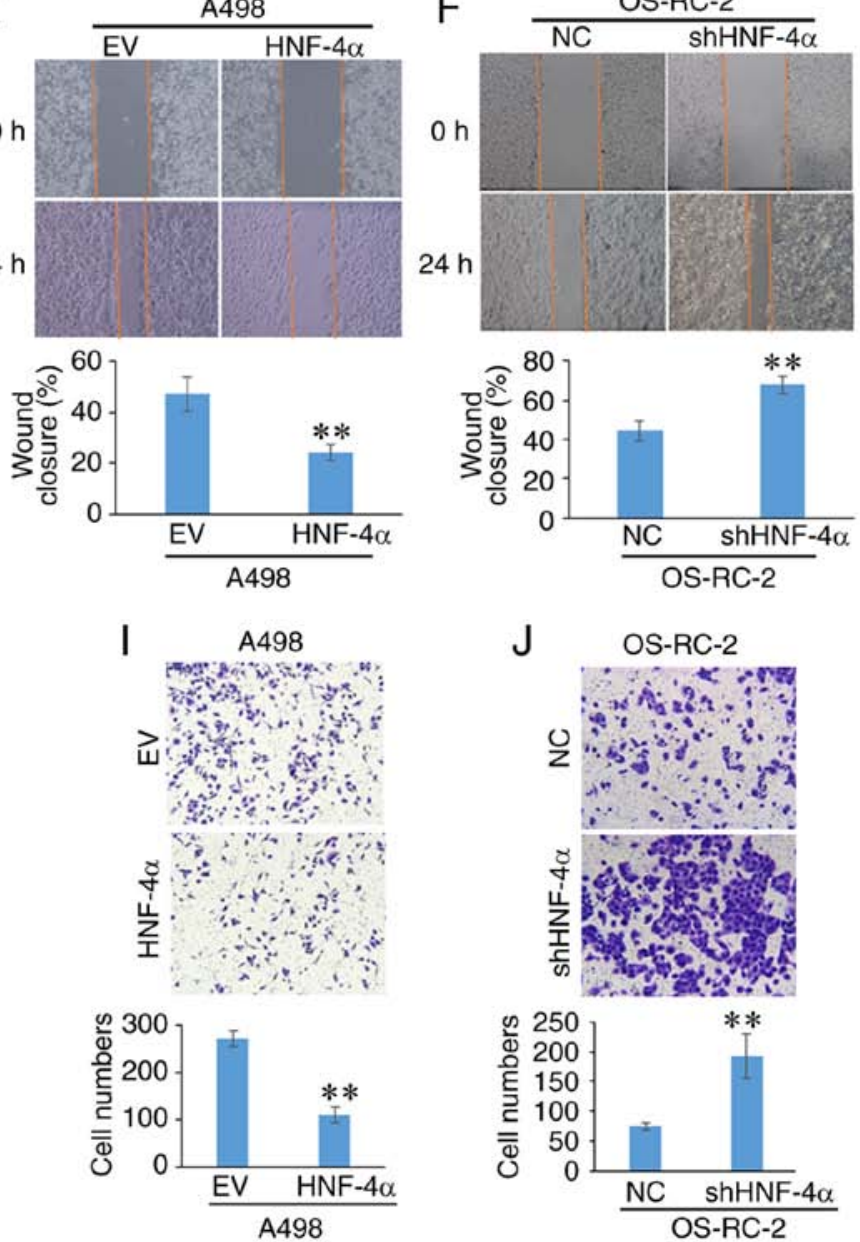

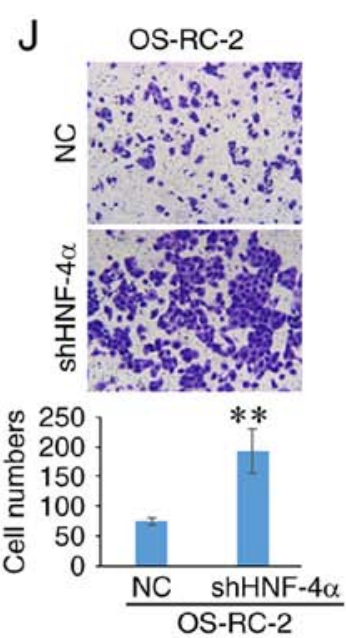

Figure 2. HNF-4 $\alpha$ regulates RCC cell migration and invasion in vitro. Protein expression of HNF-4 $\alpha$ was detected by western blotting. Actin was used as the loading control. (A) A498 cells were stably transfected with the HNF-4 $\alpha$ expression vector or EV. (B) OS-RC-2 cells were infected with retroviral vectors harboring shHNF-4 $\alpha$ or NC. (C and D) Growth curves of the indicated cells. (E and F) Representative images (magnification, x100) and quantification of the wound-healing assay in the indicated RCC cells. Representative images (magnification, x100) of (G and H) Transwell migration or (I and J) invasion assays of the indicated cells (upper panels) and statistical analyses of migratory or invasive cell numbers (lower panels). All experiments were repeated at least three times with triplicate samples. Data are presented as the mean \pm standard deviation. ${ }^{* *} \mathrm{P}<0.01$. EV, empty vector; HNF-4 $\alpha$, hepatocyte nuclear factor- $4 \alpha$; NC, non-specific control; OD, optical density; sh, short hairpin RNA.

The present study verified whether HNF-4 $\alpha$ was able to regulate cell migration and invasion via E-cadherin (Fig. 4). The expression of E-cadherin was knocked down in HNF-4 $\alpha$ overexpressing cells, and it was demonstrated that cell migration and invasion were promoted by E-cadherin knockdown (Fig. 4A, C, Eand G). In addition, E-cadherin was overexpressed in shHNF- $4 \alpha$-transfected cells, and cell invasion and migration were suppressed by E-cadherin overexpression (Fig. 4B, D, F and H). These experiments demonstrated that HNF- $4 \alpha$ may regulate cell migration and invasion through E-cadherin.

$H N F-4 \alpha$ and E-cadherin expression is positively correlated in RCC samples. The present study further explored whether the association between HNF- $4 \alpha$ and E-cadherin existed in clinical samples from patients with RCC. As determined by immunohistochemical analysis, E-cadherin expression was detected in RCC tumor and adjacent tissues. E-cadherin expression was significantly reduced in tumor tissues compared with in corresponding adjacent tissues (Fig. 5A and $\mathrm{B}$ ). In addition, lower expression of E-cadherin was demonstrated in metastatic tissues compared with in primary tumors (Fig. 5C). Furthermore, reduced HNF-4 $\alpha$ expression was frequently associated with lower E-cadherin expression, and vice versa (Fig. 5D).

Furthermore, 1,158 cases of patients with RCC in TCGA database were used to verify the association between HNF- $4 \alpha$ and E-cadherin. As shown in Fig. 5E, the mRNA expression levels of E-cadherin were decreased in tumor tissues compared with in normal tissues adjacent to tumor tissues. E-cadherin expression was also reduced in high-grade and recurrent/metastatic tumors (Fig. 5F and $\mathrm{G}$ ). In addition, there was a positive association between $\mathrm{HNF}-4 \alpha$ and E-cadherin mRNA (Fig. 5H). Compared with low-grade (I/II) RCC, HNF- $4 \alpha$ and E-cadherin expression exhibited a stronger positive correlation in high-grade (III/IV) RCC (Fig. 5I-L). Notably, low E-cadherin expression also predicted a poor prognosis in RCC (Fig. 5M and N).

\section{Discussion}

HNF- $4 \alpha$ is a liver-enriched transcription factor, which belongs to the nuclear hormone receptor superfamily. It is considered 
A
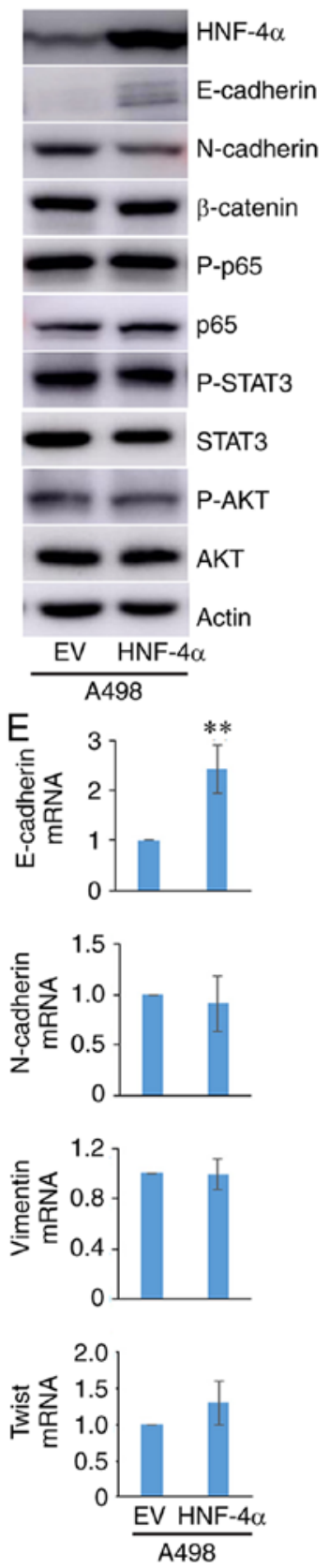

B
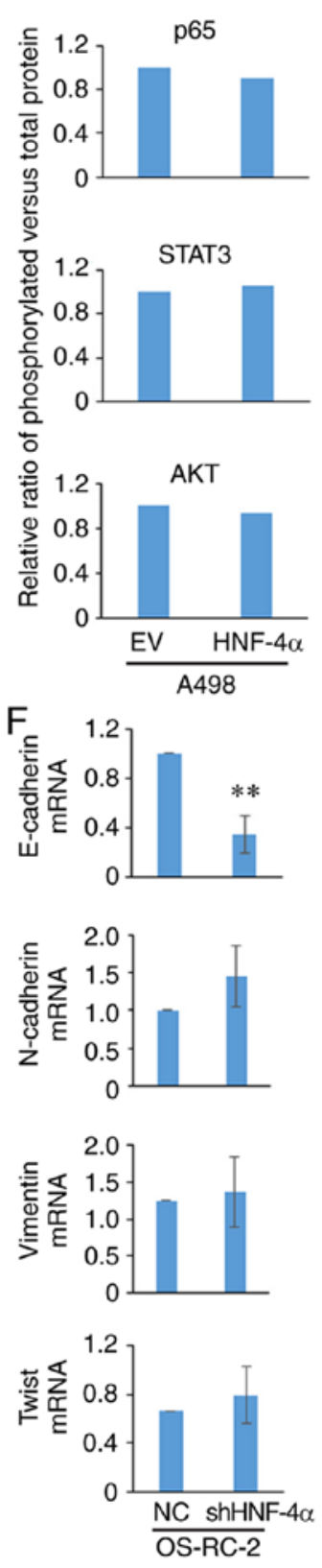

C

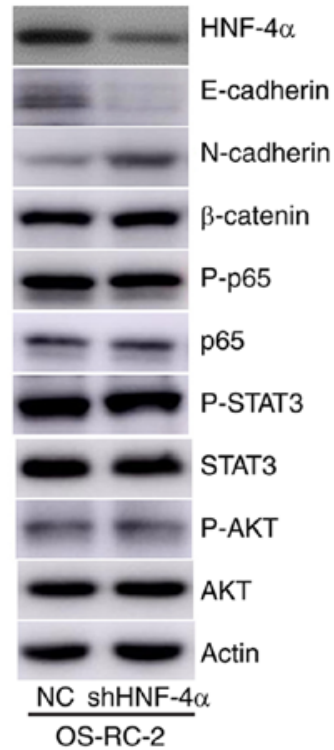

G
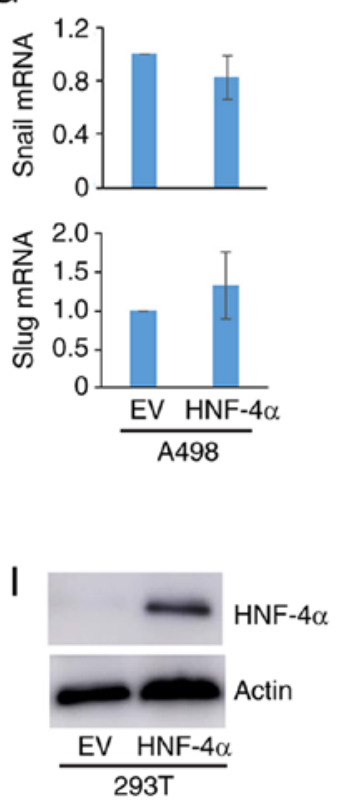

D

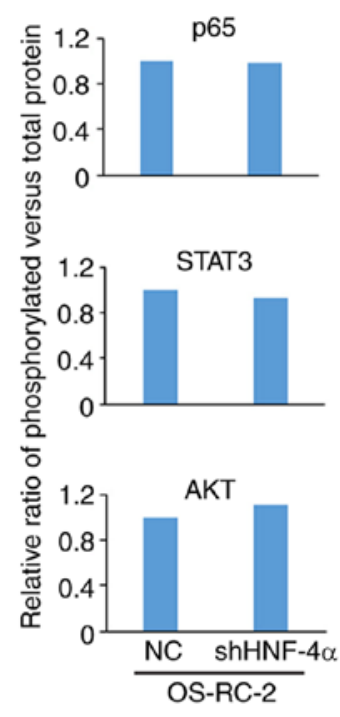

$\mathrm{H}$
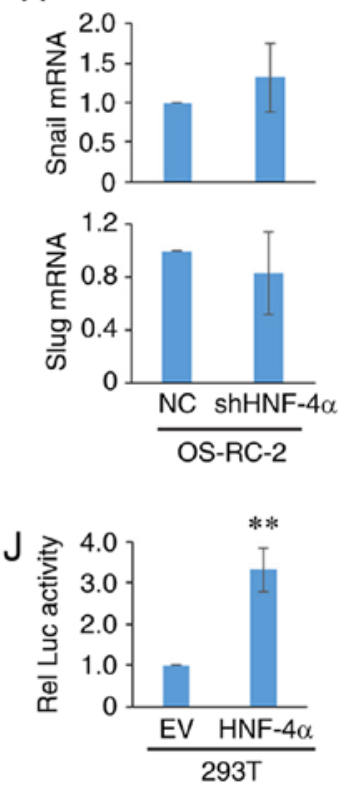

Figure 3. HNF-4 $\alpha$ regulates the expression of E-cadherin. (A and C) Expression levels of the indicated proteins were detected by western blotting. Actin was used as the loading control. (B and D) Ratio of phosphorylated protein expression versus total protein expression normalized to actin. (E-H) mRNA expression levels of the indicated genes were detected by reverse transcription-quantitative polymerase chain reaction in the indicated cells. (I and J) $293 \mathrm{~T}$ cells were transfected with luciferase reporter plasmids driven by the E-cadherin promoter with or without the HNF-4 $\alpha$ expression vector for 36 h; (I) HNF-4 $\alpha$ expression was detected by western blotting with actin as a loading control and (J) relative luciferase activity was detected. Data are presented as the mean \pm standard deviation. ${ }^{* *} \mathrm{P}<0.01$; Student's t-test. EV, empty vector; HNF-4 $\alpha$, hepatocyte nuclear factor-4 $\alpha$; NC, non-specific control; P, phosphorylated; sh, short hairpin RNA.

to be one of the central components of the HNF regulatory network in hepatic cells. Substantial evidence has revealed that HNF- $4 \alpha$ is downregulated in human hepatocellular carcinoma (HCC) tissues compared with in adjacent noncancerous tissues, and that restoration of $\mathrm{HNF}-4 \alpha$ function may induce differentiation of hepatoma cells into hepatocytes (8). Recently, HNF- $4 \alpha$ introduction was revealed to not only be able to attenuate liver fibrosis and cirrhosis, but also completely block hepatocarcinogenesis (26). Furthermore, downregulation of HNF- $4 \alpha$ in HCC results in loss of epithelial morphology, dedifferentiation, and acquirement of the ability to invade and metastasize, which implies that HNF- $4 \alpha$ may be a novel therapeutic target for $\mathrm{HCC}(8)$. The present study revealed that HNF- $4 \alpha$ was downregulated in RCC and its downregulation was associated with a poor prognosis in patients with RCC. In addition, knockdown of HNF- $4 \alpha$ expression significantly promoted the migration and invasion of RCC cells. These results suggested that $\mathrm{HNF}-4 \alpha$ may function as a tumor suppressor in the development of RCC. There is no doubt that inactivation or downregulation of $\mathrm{HNF}-4 \alpha$, which confers a number of selective advantages to the tumor, is a frequent event during tumor progression and is associated with a more 
A

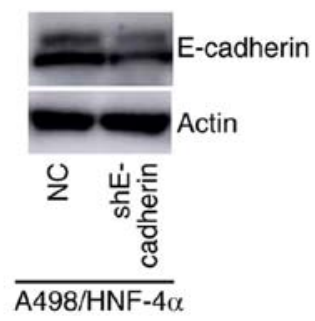

B

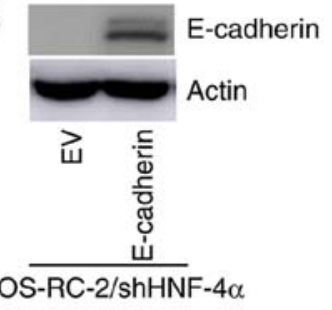

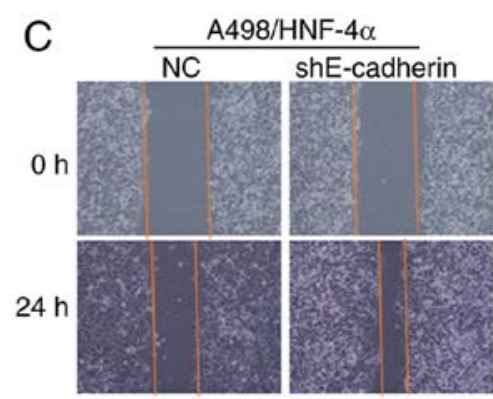

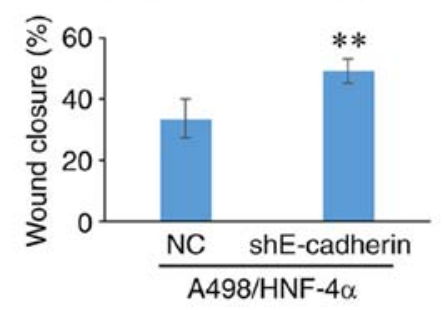

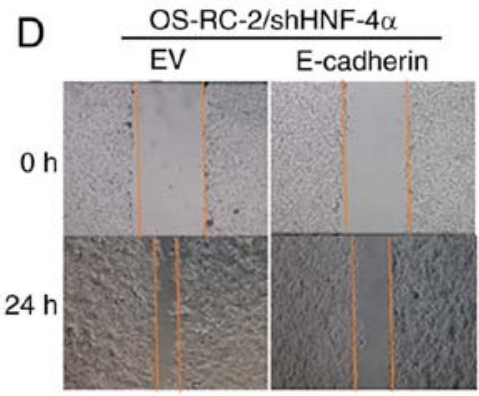

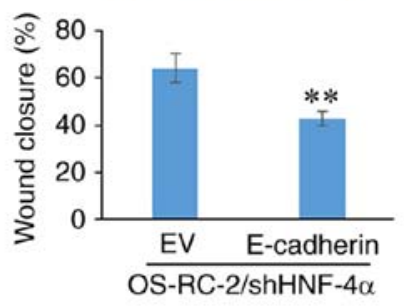

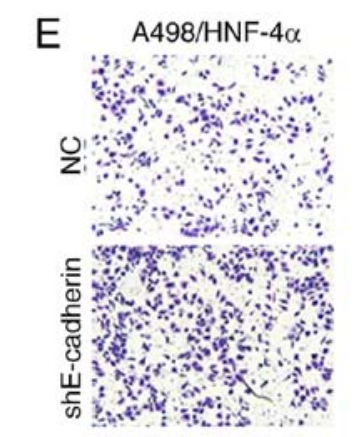
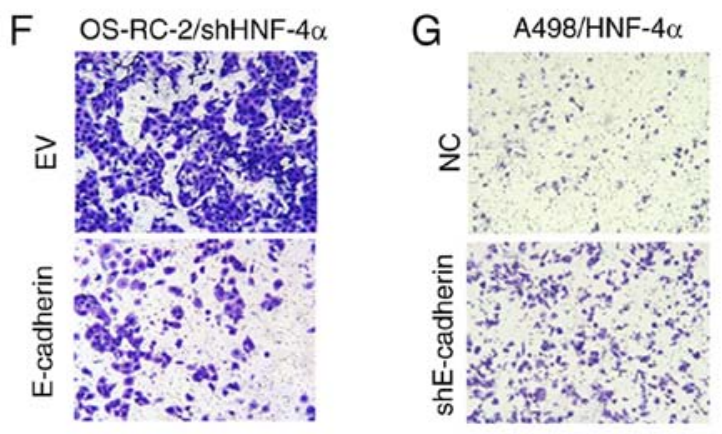

H OS-RC-2/shHNF-4a
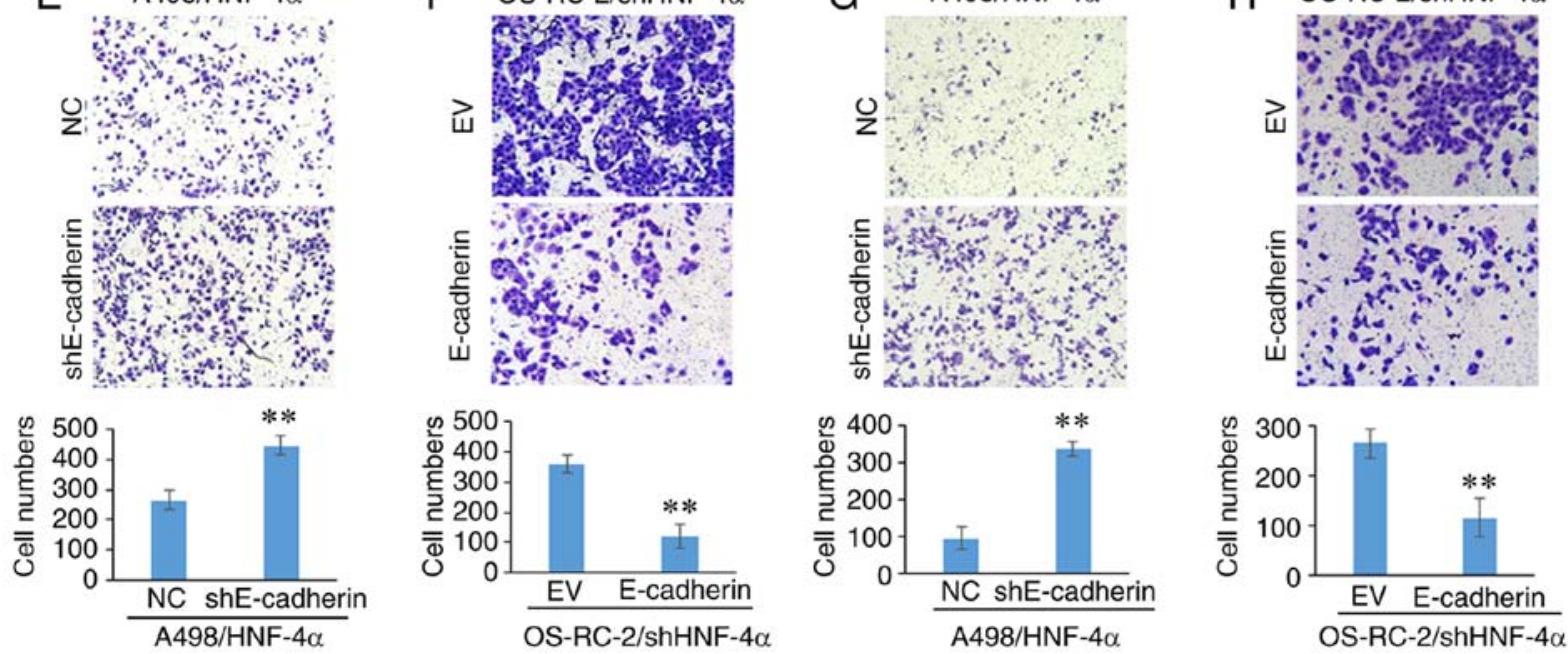

Figure 4. E-cadherin regulates the migration and invasion of RCC cells. The expression of E-cadherin was detected by western blotting; actin was used as the loading control. (A) A498/HNF-4 $\alpha$ cells were stably transfected with shE-cadherin or NC. (B) OS-RC-2/shHNF-4 $\alpha$ cells were infected with an E-cadherin expression vector or EV. (C and D) Representative images (magnification, x100) and quantification of the wound-healing assay in the indicated RCC cells. Representative images (magnification, x100) of Transwell (E and G) migration or ( $F$ and $H$ ) invasion assays of the indicated cells (upper panels), and statistical analyses of migratory or invasive cell numbers (lower panels). All experiments were repeated at least three times with triplicate samples. Data are presented as the mean \pm standard deviation. ${ }^{* *} \mathrm{P}<0.01$; Student's t-test. EV, empty vector; HNF-4 $\alpha$, hepatocyte nuclear factor-4 $\alpha$; NC, non-specific control; sh, short hairpin RNA.

malignant tumor phenotype. These findings suggested that HNF-4 $\alpha$ may be considered a candidate tumor suppressor in tissue types where it normally serves an important role. Whether HNF-4 $\alpha$ can be used as a prognostic indicator or a drug target is worth further exploration.

E-cadherin expression may be regulated by various transcription factors, including Snail and Slug, which belong to the Snail family. Snail and Slug are able to bind to the promoter and directly inhibit the transcription of E-cadherin (27-29). Recently, the oncogenic serine/threonine kinase AKT has been reported to suppress transcription of E-cadherin (24). Furthermore, E-cadherin may be a direct transcriptional target of HNF-4 $\alpha$ in HCC (30). The present results confirmed that downregulation of E-cadherin in RCC may be mediated by HNF-4 $\alpha$. Notably, a strong correlation was determined between the expression of HNF- $4 \alpha$ and E-cadherin in high-grade RCC cases, suggesting that regulation of
E-cadherin by HNF- $4 \alpha$ may be closely associated with the malignancy of RCC. As a transcription factor, whether $\mathrm{HNF}-4 \alpha$ serves other roles in the occurrence, progression and treatment of RCC, and its molecular mechanism are also worthy of further study.

In conclusion, the present results demonstrated that HNF-4 $\alpha$ was downregulated and associated with a poor prognosis in RCC. Low HNF- $4 \alpha$ expression promoted cell invasion and migration in RCC. In addition, E-cadherin may serve an important role in the process through which $\mathrm{HNF}-4 \alpha$ regulates tumor invasion and migration. Unfortunately, we failed to establish a mouse model of RCC metastasis; further studies are required to explore whether $\mathrm{HNF}-4 \alpha$ regulates tumor metastasis in vivo. Taken together, the present findings provided a novel insight into the tumor suppressor role of HNF- $4 \alpha$ in RCC progression and thus may help identify novel approaches for the treatment of RCC. 
A Normal tissues

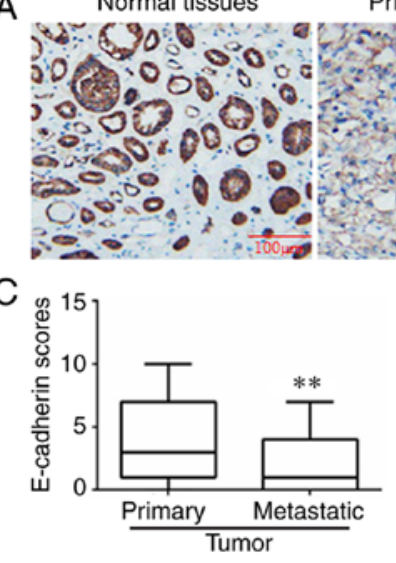

F

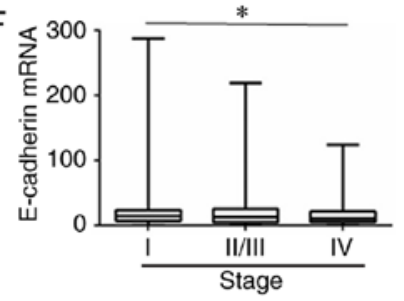

$\left.\varangle^{1000}\right] \quad P=0.0208 \mathrm{Stage} \mathrm{I}=-0.1063$

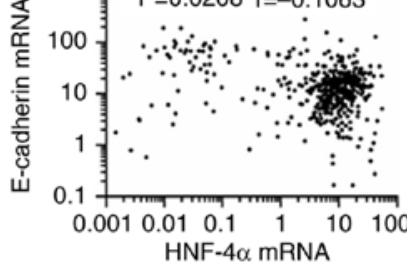

L

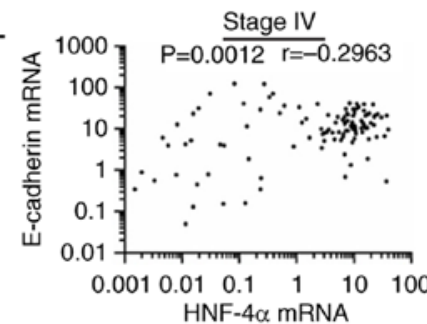

Primary tumor

Metastatic tumor

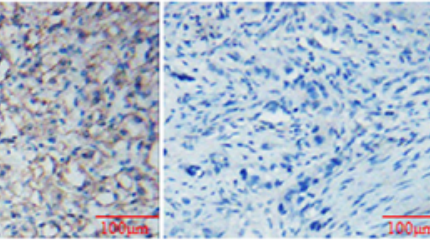

D
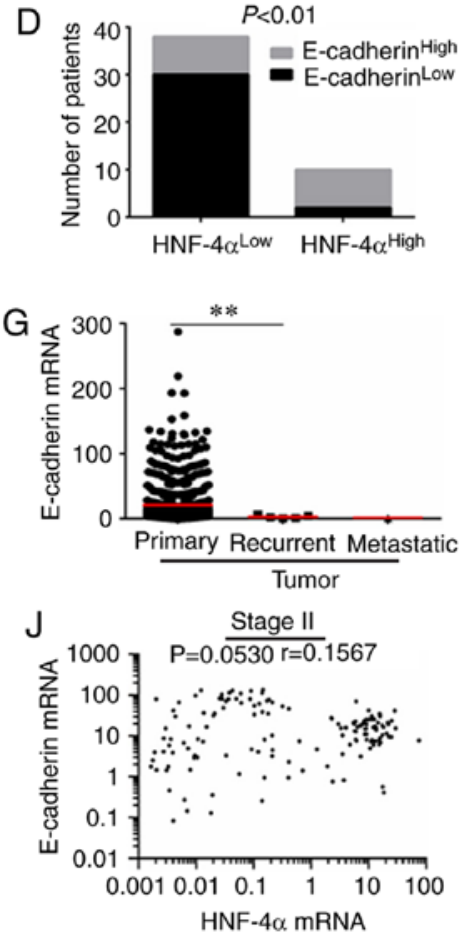

M

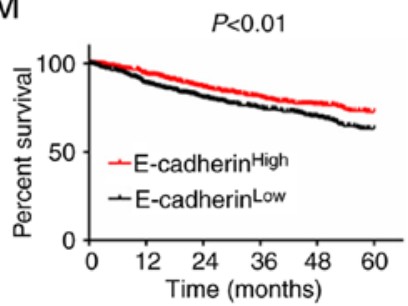

B

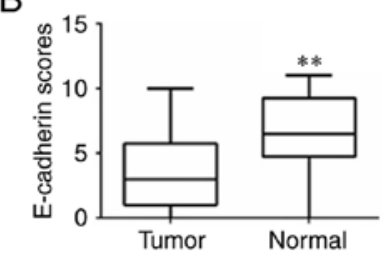

E

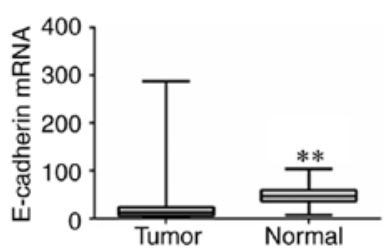

$\mathrm{H}$
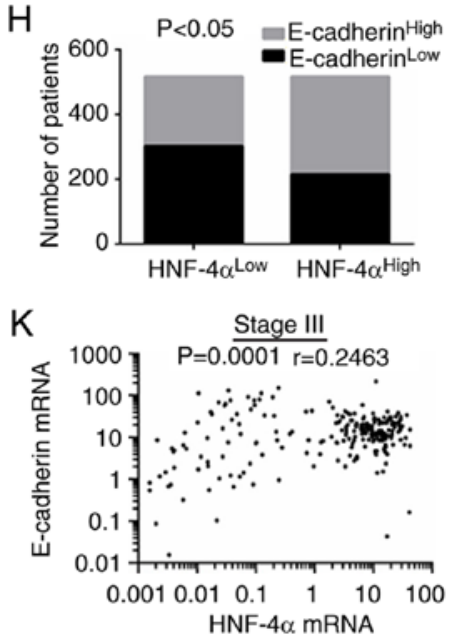

$\mathrm{N}$

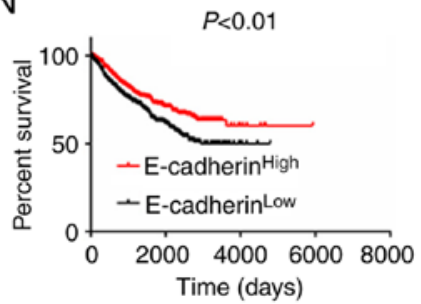

Figure 5. Expression of E-cadherin and correlation analysis in RCC. (A) Representative IHC images of RCC for E-cadherin expression. (B and C) E-cadherin expression scores are illustrated as box plots. (D) Analysis of HNF-4 $\alpha$ and E-cadherin protein expression, calculated via Pearson's $\chi^{2}$ test. (E-G) E-cadherin expression in different pathological types and grades of RCC, derived from TCGA database. (E) Tumor $(\mathrm{n}=1,019)$ and normal tissues $(\mathrm{n}=139)$ were compared and calculated via Student's t-test. (F) Stage I $(n=473)$, II/III $(n=390)$ and IV ( $n=117)$ RCC tissues were compared and calculated via one-way ANOVA followed by Bonferroni post hoc test. (G) Primary $(n=1,013)$, recurrent $(n=5)$ and metastatic $(n=1)$ RCC tissues were compared and calculated via one-way ANOVA followed by Bonferroni post hoc test. (H) Analysis of HNF- $4 \alpha$ and E-cadherin mRNA expression, calculated via Pearson's $\chi^{2}$ test. (I-L) Correlation analysis of HNF- $4 \alpha$ and E-cadherin mRNA expression in different grades of RCC, calculated via Spearman's rank correlation. (M) Association between E-cadherin expression and 5-year survival rate of patients with RCC, according to TCGA database. Data were analyzed using log-rank test, $\mathrm{P}<0.01$. (N) Overall survival of patients with RCC with high or low expression of E-cadherin; data were derived from TCGA database. Data were analyzed using log-rank test, $\mathrm{P}<0.01$. ${ }^{*} \mathrm{P}<0.05,{ }^{* *} \mathrm{P}<0.01$. HNF-4 $\alpha$, hepatocyte nuclear factor- $4 \alpha$; RCC, renal cell carcinoma; TCGA, The Cancer Genome Atlas.

\section{Acknowledgements}

The authors would like to thank Dr Yong Zhang (Rui-Jin Hospital) and Dr Shaoming Shen (Shanghai Jiaotong University) for providing cells and vectors.

\section{Funding}

This work was supported by the National Natural Science Foundation of China (grant nos. 81502197, 81472501 and 81772849) and the Fundamental Research Funds for the Central Universities (grant no. 2016KJ050).

\section{Availability of data and materials}

The datasets used and/or analyzed during the current study are available from the corresponding author on reasonable request.

\section{Authors' contributions}

QW and $X Y$ designed the experiments and wrote the manuscript. YG, YY, JG and QZ performed the majority of the experiments and conducted the data analysis. DB, FW, ZC and LL participated in sample collection and other experiments. 


\section{Ethics approval and consent to participate}

The present study was approved by the Medical Ethical Committee of Shanghai Tenth People's Hospital. All patients provided written informed consent prior to participation in the present study based on the guidelines of the Medical Ethical Committee of Shanghai Tenth People's Hospital prior to participating in the present study.

\section{Patient consent for publication}

All patients provided written informed consent before participation and agreed to publication of the present study.

\section{Competing interests}

The authors declare that they have no competing interests.

\section{References}

1. Erickson LA: Clear cell renal cell carcinoma. Mayo Clin Proc 93 813-814, 2018

2. Ljungberg B, Bensalah K, Canfield S, Dabestani S, Hofmann F, Hora M, Kuczyk MA, Lam T, Marconi L, Merseburger AS, et al: EAU guidelines on renal cell carcinoma: 2014 update. Eur Urol 67: 913-924, 2015.

3. Siegel RL, Miller KD and Jemal A: Cancer statistics, 2016. CA Cancer J Clin 66: 7-30, 2016.

4. Kaelin WG Jr: Treatment of kidney cancer: Insights provided by the VHL tumor-suppressor protein. Cancer 115 (Suppl 10) S2262-S2272, 2009

5. Gao YH, Wu ZX, Xie LQ, Li CX, Mao YQ, Duan YT, Han B, Han SF, Yu Y, Lu HJ, et al: VHL deficiency augments anthracycline sensitivity of clear cell renal cell carcinomas by down-regulating ALDH2. Nat Commun 8: 15337, 2017.

6. Ljungberg B, Campbell SC, Choi HY, Jacqmin D, Lee JE, Weikert $S$ and Kiemeney LA: The epidemiology of renal cell carcinoma. Eur Urol 60: 615-621, 2011.

7. Chen S, Wang Q, Wang L, Chen H, Gao X, Gong D, Ma J, Kubra S, Yao X, Li X, et al: REGgamma deficiency suppresses tumor progression via stabilizing CK1epsilon in renal cell carcinoma. Cell Death Dis 9: 627, 2018.

8. Lazarevich NL and Fleishman DI: Tissue-specific transcription factors in progression of epithelial tumors. Biochemistry (Mosc) 73: 573-591, 2008.

9. Morimoto A,Kannari M, Tsuchida Y,Sasaki S, Saito C,Matsuta T, Maeda T, Akiyama M, Nakamura T, Sakaguchi M, et al: An HNF4alpha-microRNA-194/192 signaling axis maintains hepatic cell function. J Biol Chem 292: 10574-10585, 2017.

10. Battle MA, Konopka G, Parviz F, Gaggl AL, Yang C, Sladek FM and Duncan SA: Hepatocyte nuclear factor 4alpha orchestrates expression of cell adhesion proteins during the epithelial transformation of the developing liver. Proc Natl Acad Sci USA 103: 8419-8424, 2006.

11. Wang Z and Burke PA: Hepatocyte nuclear factor-4alpha interacts with other hepatocyte nuclear factors in regulating transthyretin gene expression. FEBS J 277: 4066-4075, 2010.

12. Stegmann A, Hansen M, Wang Y, Larsen JB, Lund LR, Ritié L, Nicholson JK, Quistorff B, Simon-Assmann P, Troelsen JT and Olsen J: Metabolome, transcriptome, and bioinformatic cis-element analyses point to HNF-4 as a central regulator of gene expression during enterocyte differentiation. Physiol Genomics 27: 141-155, 2006.

13. Hayhurst GP, Strick-Marchand H, Mulet C, Richard AF, Morosan S, Kremsdorf D and Weiss MC: Morphogenetic competence of HNF4 alpha-deficient mouse hepatic cells. J Hepatol 49: 384-395, 2008.
14. Lazarevich NL, Shavochkina DA, Fleishman DI, Kustova IF, Morozova OV, Chuchuev ES and Patyutko YI: Deregulation of hepatocyte nuclear factor 4 (HNF4) as a marker of epithelial tumors progression. Exp Oncol 32: 167-171, 2010.

15. Lazarevich NL, Cheremnova OA, Varga EV, Ovchinnikov DA, Kudrjavtseva EI, Morozova OV, Fleishman DI, Engelhardt NV and Duncan SA: Progression of HCC in mice is associated with a downregulation in the expression of hepatocyte nuclear factors. Hepatology 39: 1038-1047, 2004.

16. Sel S, Ebert T, Ryffel GU and Drewes T: Human renal cell carcinogenesis is accompanied by a coordinate loss of the tissue specific transcription factors HNF4 alpha and HNF1 alpha. Cancer Lett 101: 205-210, 1996.

17. van Staalduinen J, Baker D, Ten Dijke P and van Dam H: Epithelial-mesenchymal-transition-inducing transcription factors: New targets for tackling chemoresistance in cancer? Oncogene 2018 (Epub ahead of print).

18. Devlin JR and Verschuren EW: More than a tumor suppressor: E-cadherin loss drives lung cancer metastasis. Am J Respir Cell Mol Biol 59: 141-142, 2018.

19. Kourtidis A, Lu R, Pence LJ and Anastasiadis PZ: A central role for cadherin signaling in cancer. Exp Cell Res 358: 78-85, 2017.

20. Livak KJ and Schmittgen TD: Analysis of relative gene expression data using real-time quantitative PCR and the 2(-Delta Delta C(T)) method. Methods 25: 402-408, 2001.

21. Shen SM, Guo M, Xiong Z, Yu Y, Zhao XY, Zhang FF and Chen GQ: AIF inhibits tumor metastasis by protecting PTEN from oxidation. EMBO Rep 16: 1563-1580, 2015.

22. Yu D, An X, Fan W, Wang X, He Y and Li B: PNUTS mediates ionizing radiation-induced CNE-2 nasopharyngeal carcinoma cell migration, invasion, and epithelial-mesenchymal transition via the PI3K/AKT signaling pathway. Onco Targets Ther 12: 1205-1214, 2019

23. Min HJ, Lee Y, Zhao XF, Park YK, Lee MK, Lee JW and Kim S: TMPRSS4 upregulates uPA gene expression through JNK signaling activation to induce cancer cell invasion. Cell Signal 26: 398-408, 2014.

24. Li X, Bao C, Ma Z, Xu B, Ying X, Liu X and Zhang X: Perfluorooctanoic acid stimulates ovarian cancer cell migration, invasion via ERK/NF-кB/MMP-2/-9 pathway. Toxicol Lett 294: 44-50, 2018.

25. Yao HH, Zhao YJ, He YF, Huang DB and Wang W: Knockdown of AGGF1 inhibits the invasion and migration of gastric cancer via epithelial-mesenchymal transition through Wnt/beta-catenin pathway. Cancer Cell Int 19: 41, 2019.

26. Chang Z, Cai C, Han D, Gao Y, Li Q, Feng L, Zhang W, Zheng J, Jin J, Zhang $\mathrm{H}$ and Wei Q: Anoctamin5 regulates cell migration and invasion in thyroid cancer. Int J Oncol 51: 1311-1319, 2017.

27. Batlle E, Sancho E, Franci C, Domínguez D, Monfar M, Baulida J and García De Herreros A: The transcription factor snail is a repressor of E-cadherin gene expression in epithelial tumour cells. Nat Cell Biol 2: 84-89, 2000.

28. Prokop JW, Liu Y, Milsted A, Peng H and Rauscher FJ III: A method for in silico identification of SNAIL/SLUG DNA binding potentials to the E-box sequence using molecular dynamics and evolutionary conserved amino acids. J Mol Model 19: 3463-3469, 2013.

29. Moreno-Bueno G, Cubillo E, Sarrio D, Peinado H, Rodríguez-Pinilla SM, Villa S, Bolós V, Jordá M, Fabra A, Portillo F, et al: Genetic profiling of epithelial cells expressing E-cadherin repressors reveals a distinct role for Snail, Slug, and E47 factors in epithelial-mesenchymal transition. Cancer Res 66: 9543-9556, 2006.

30. Spath GF and Weiss MC: Hepatocyte nuclear factor 4 provokes expression of epithelial marker genes, acting as a morphogen in dedifferentiated hepatoma cells. J Cell Biol 140: 935-946, 1998.

This work is licensed under a Creative Commons Attribution-NonCommercial-NoDerivatives 4.0 International (CC BY-NC-ND 4.0) License. 